\title{
Multi-aperture interferometry at Concordia
}

\section{Eric Fossat ${ }^{1}$, Farrokh Vakili $^{1}$, Eric Aristidi ${ }^{1}$, Bruno Lopez ${ }^{2}$, François-Xavier Schmider ${ }^{1}$, Karim Agabi $^{1}$, Jean-Baptiste Daban ${ }^{1}$, Fatmé Allouche ${ }^{1}$, Adrian Belu ${ }^{1}$, Pierre-Marie Gori ${ }^{1}$, Géraldine Guerri $^{1}$ and Bruno Valat ${ }^{1}$}

${ }^{1}$ LUAN, UMR 6525, Université de Nice - Sophia Antipolis, Parc Valrose, F-06108 Nice, France email: eric.fossat@unice.fr

${ }^{2}$ Observatoire de la cote d'Azur, BP 4229, F-06304 Nice, France

\section{Introduction}

The next generation (post-VLTI) of multi-telescope interferometric arrays operated in optical/infrared wavelengths should be kilometric, from 1 to $10 \mathrm{~km}$. The Concordia station offers a unique opportunity to set such an interferometer in the best atmospheric conditions presently known on Earth.

\section{KEOPS - the concept}

The Dome C site astronomical qualities begin to be well bracketed. After several summers and now almost two winter-over site testing campaigns, it is clear that it is, for many astronomical parameters, the best, or one of the very best sites on Earth. Some of these parameters still demand additional investigation or more statistics. But the global quality has been proved to be enough out of range for attracting an ever increasing scientific community. French and Italian funding has started to be raised, so that beyond the site testing, real astronomical programmes are expected to be operated in 2008 (IRAIT from Italy and A-STEP from France).

On the longer term range, medium and far infrared imaging on one hand, and on the other hand Extremely High Resolution Imaging even in visible light are among the favorite targets in the prospect studies. Some are thinking of an Antarctic ELT, to be set above the 30-m turbulent surface boundary layer, others would prefer an multi mirror interferometer. Such an interferometer, that could be called an optical equivalent to the VLA in radio waves or ALMA in millimetric, can possibly be regarded as the next generation, post-VLTI, of large size optical interferometry.

Of course, optical long baseline imaging interferometry is extremely difficult, as the technical challenges go more or less as the inverse of the wavelength, and that means a factor 100 to 1000 for optical or near-IR as compared to the millimetric case of ALMA. However and to some extent, it can now be regarded as a mature observing technique. Several optical arrays are able to provide 2-D maps: NPOI in Arizona, COAST at Cambridge, UK, CHARA at Mount Wilson, California and of course the VLTI at Paranal, Chile. At the 2004 Liège International Astrophysical Colloquium devoted to the Science case for next generation optical/infrared interferometric facilities (the post-VLTI era), it was recognised by Pierre Lena that on one hand "the next interferometer generation should operate at least from 1 to $12 \mu \mathrm{m}$ and have kilometric baselines (1 to $10 \mathrm{~km}$ at most)", and on the other hand "the Dome Csite characteristics, as far as they are known today, appear to be of an entirely different class than any other ground-based site: in fact, this site classifies as an intermediate one between space and conventional ground. 
They appear especially favorable for interferometry (transparency, isoplanetism, stability of the atmosphere, area)".

The concept of KEOPS results from these statements. It emerges as an imaging array of optical diffraction limited telescopes of 1.5 to $2-\mathrm{m}$ diameter in Dome $\mathrm{C}$ conditions. These telescopes are spread over three concentric rings of 200, 348 and 676 meter radii. Six or seven telescopes on the first ring, 12 or 13 on the second, 18 or 19 on the outer one. These numbers offer optimized $u-v$ coverage to achieve a 1 mas resolution at $10 \mu \mathrm{m}$ in order to resolve the angular distance between a star and its exo-Earth at a one kpc distance. KEOPS is an implicitly co-phased array operated in the so-called hypertelescope mode (Labeyrie et al. 2003), but using a more efficient nulling design named IRAN (Vakili et al. 2004). KEOPS has an equivalent collecting surface comparable to the Keck interferometer, but located in extreme cold, dry and excellent seeing conditions of the Antarctica plateau. It will challenge a $30 \mathrm{~m}$-class ELT, and the number of available square kilometers on the polar plateau is essentially unlimited!

\section{KEOPS - the science rationale}

The bottom line of an interferometric instantaneous field of view is the Airy disc of individual telescopes. Considering the 1.5 to 2 -m diameter proposed for KEOPS, one may expect a sub-mas resolution across a 1 arcsec field of view. Thus, unlike classical wide field telescopes, KEOPS offers Ultra High Spatial Resolution imaging with a reasonable wide field of $2000 \times 2000$ resolution elements (resels). The inefficient filling factor (less than $10^{-3}$ ) could be compensated by Earth rotation synthesis for imaging compact objects which benefit from the long polar night of Antarctica. Beyond the search for exo-Earths, it could bring significant breakthroughs in the study of galactic and extra-galactic objects from the visible to the thermal infrared wavelenths inaccessible from any other ground based site. That could be stellar surface imaging, the central engines of YSO's, the cores of AGN, or even the ballet of stars rotating around the central black hole of galaxies as far as a few millions light-years. In fact the number of possible scientific scoops with such an instrument is nearly infinite.

\section{References}

Labeyrie, A., Le Coroller, H., Dejonghe, J., et al. 2003, SPIE, 4852, 236

Vakili, F., Aristidi, E., Abe, L., \& Lopez, B. 2004, A\&A A, 421, 147 